\title{
3-D Structure of Z-disks Isolated from the Flight Muscle of Lethocerus indicus
}

Fatemeh Abbasi Yeganeh ${ }^{1}$, Corinne Summerill ${ }^{2}$, Dianne W. Taylor ${ }^{1}$, Hamidreza Rahmani ${ }^{1}$, Kenneth A. Taylor ${ }^{1 *}$

1. Institute of Molecular Biophysics, Florida State University, Tallahassee, FL, USA.

2. Department of Biological Science, Florida State University, Tallahassee, FL, USA.

* Corresponding author: taylor@bio.fsu.edu

The reconstruction of the recent publication of a 3-D image of isolated Z-disk from Apis mellifera flight muscle [1] is the first of its kind utilizing isolated Z-disks, confirmed many details of the previously published 3-D image obtained from plastic sections. However, they used relatively harsh treatment of $\mathrm{KCl} / \mathrm{KI}$ to remove both thick and thin filaments in a single step that regardless produced a specimen suitable for cryoelectron tomography. Here, we use a different approach for Z-disk isolation from flight muscle from the large waterbug Lethocerus indicus that combines $1.4 \mathrm{M} \mathrm{NaCl}$ and $10 \mathrm{mM}$ pyrophosphate to remove the thick filaments and gelsolin to remove the thin filaments. Myofibrils are suspended in the high salt-pyrophosphate buffer. I-Z-I brushes are then centrifuged gently to remove the dissolved thick filaments. The I-Z-I brushes are placed on the grid bar side of a Quantifoil ${ }^{\mathrm{TM}}$ grid and treated with a calcium insensitive gelsolin construct for $4.5 \mathrm{~min}$ after which the broken up thin filaments are washed from the grid and the $3 \mu \mathrm{m}$ diameter Z-disk are visualized over holes. Tilt series are collected on a Titan Krios electron microscope, with a DE-20 camera and merged using PROTOMO [6]. Fourier transforms of the Z-disks show spots from a hexagonal lattice with a spacing of $520 \AA$ extending to $87 \AA$.

The tomograms are similar to those from honey bee with some differences. The large solvent channels of Apis are not as prominent in Lethocerus Z-disks (Fig. 3) and this is reflected in weak to absent intensity in the first order diffraction from the hexagonal lattice (Fig. 1). We conclude Z-disks from Apis mellifera and Lethocerus indicus are similar but not identical. In hexagonal lattice arrangement of thin filaments, each unit cell contains six thin filaments, three filaments each with opposite orientations. Filaments of opposite orientation overlapped for a distance of $\sim 87 \mathrm{~nm}$, which is very close to the $\sim 80 \mathrm{~nm}$ overlap found in Apis Z-Discs [2] (Fig. 2). Symmetrized global average revealed the unique network of connecting densities that maintains the arrangement of the thin filaments of opposite and same orientations. The thin filament connections form two different channels: the large channel formed by the thin filaments at the apexes of each unit cell that are formed by six thin filaments of altering orientation, and the two small solvent channels within the unit cell that are formed by three thin filaments of the same polarity (Fig. 3). There is ample evidence that the actin filaments in the overlap zone of both bee and waterbug flight muscle present a helical array of target zones around each thick filament. However, Squire [5] has pointed out that Cheng and Dethearage's 3D reconstruction of the honey bee Z-disk, when extrapolated into the Aband, implies rings of target zones rather than helices of target zones surrounding the thick filament. This discrepancy might be resolved if there were 3-fold screw axes at the lattice and trigonal positions of the of the insect Z-band instead of three-fold rotation axes; thus making the insect Z-band P ${ }_{1} 21$, instead of P321 assumed previously [2]. The A-band lattice of Lethocerus flight muscle has six actin filaments surrounding a particular myosin filament and these present a helical array of actin 'target' areas $[4,5]$ for the cross-bridges on the myosin filament. We aim to resolve the actin structure in Z-disc at sufficient resolution to find an answer to this problem. 
References:

[1] J Rusu et al., J. Muscle Res. Cell Motil. 38 (2017), p. 241.

[2] N Cheng and JE Deatherage, J. Cell Biol. 108 (1989), p. 1761.

[3] J Cope, J Heumann, \& A Hoenger, Curr. Protoc. Protein Sci., ch 17 (2011), Unit17.13.

[4] MK Reedy, J. Molec. Biol. 31 (1968), p. 155.

[5] JM Squire, J. Molec. Biol. 72 (1972), p. 125.

[6] H Winkler and KATaylor, Ultramicroscopy 106 (2006), p. 240.

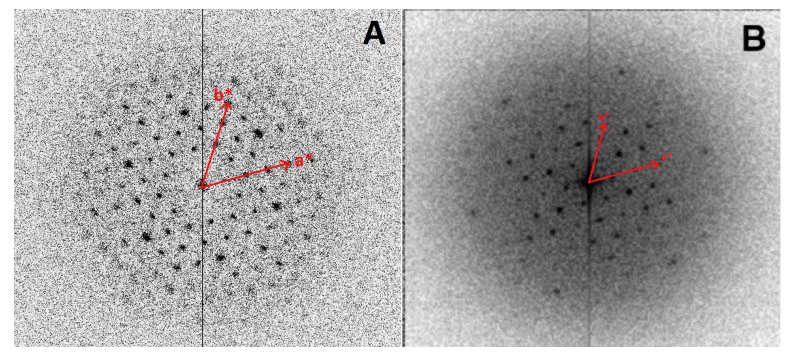

Figure 1. Comparison between Apis and Lethocerus Fourier Transforms. A) Fourier transform of cryo-EM image of a Lethocerus Z-disc which extends to 6 orders. A repeating lattice is demonstrable in the Fourier transform. The 1,0 lattice spot is weak to absent. B) Fourier transform Z-disc from Honeybee flight muscle with strong first order diffraction [1].

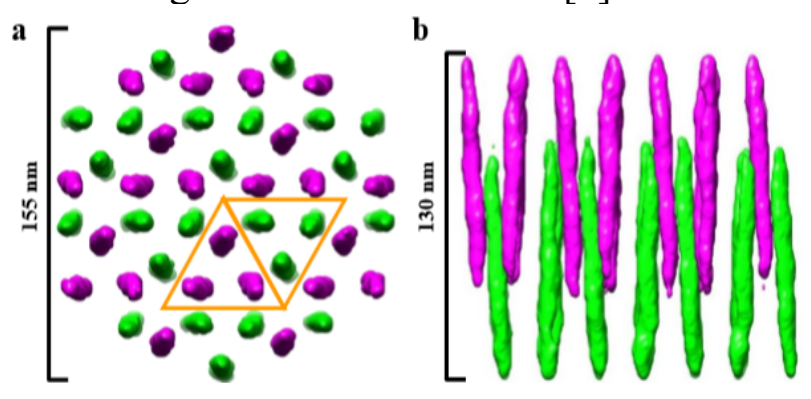

Figure 2. Filament Arrangement in Lethocerus Z-Disc. This image obtained after subvolume averaging and symmetrization and visualized by Chimera. a) Top view of flight muscle Z-disc $(\mathrm{z}=0)$. b) The Z-Disc viewed from the side.
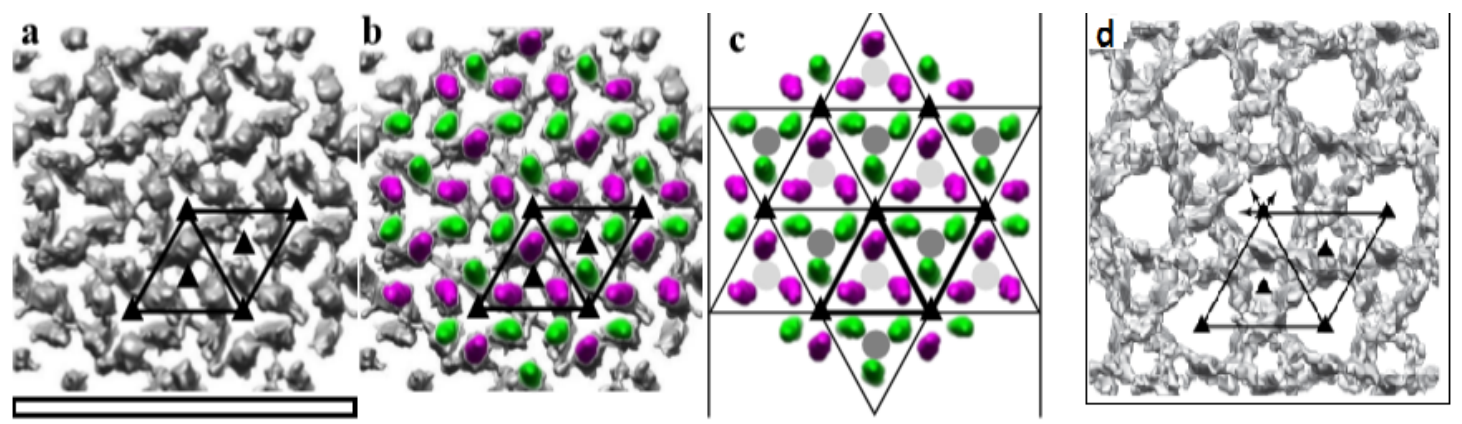

Figure 3. Density Map of Global Averages of Lethocerus Z-disc. a) Density map (grey) of a symmetrized global average showing the connecting densities that form the Z-Disc. b) Overlap of thin filaments from Figure 2 (purple and green) on the density map. c) The p321 symmetry that is seen in the thin filament arrangement. The dark and light grey circles represent the positions of the thick filaments in the A-band of adjacent sarcomeres. The unit cell in all the images are represented with thick black lines. White scale bar $=155 \mathrm{~nm}$. d) Density map showing the connecting densities that form the Z-Disc from Apis [1] showing channels of distinctly different sizes compared to Lethocerus. 